\title{
EFEITOS DA INTERVENÇÃO AQUÁTICA SOBRE A MOBILIDADE EM IDOSOS SEDENTÁRIOS DA COMUNIDADE - RESULTADOS PRELIMINARES
}

Daniela Lemes Ferreira - UFMS - daniela_lemes@hotmail.com Dayane Melo Campos - UFMS - dayanecampos2824@gmail.com Nathany C da Silva - UFMS - nathany_clara@ufms.br Ana Luísa Janducci - UFSCAR - ajanducci@gmail.com Gustavo Christofoletti - UFMS - g.chistofoletti@ufms.br Juliana Hotta Ansai - UFSCAR - jhansai@ufscar.br

\section{RESUMO}

Introdução: A intervenção aquática pode ser um ótimo recurso para melhorar mobilidade funcional nos idosos. Objetivo: Verificar os efeitos da intervenção aquática sobre a mobilidade em idosos sedentários da comunidade. Métodos: Trata-se de dados parciais de um ensaio clínico controlado randomizado, unicêntrico, em idosos da comunidade. Os idosos sedentários foram divididos aleatoriamente em Grupo Controle (GC) e Grupo Intervenção (GI). No GI, foi realizado um programa de hidroterapia por 16 semanas (1 hora de sessão, 2xsemana, progressão individualizada), que incluiu: aquecimento, exercícios de endurance e resistência muscular, equilíbrio, resistência aeróbica e desaquecimento, associados a tarefas cognitivas. Os participantes foram avaliados no momento inicial e após 16 semanas, por meio de anamnese e mobilidade (teste Timed up and Go (TUG) simples e associado com tarefa cognitiva-motora (TUG-DT)). Os dados foram analisados por meio do Software SPSS 20.0. Resultados: 11 idosos participaram até o momento, 6 no GI e 5 no GC. Não houve diferenças significativas nas características clínicas e sociodemográficas iniciais entre grupos, exceto no sexo, com prevalência maior de mulheres no GI. Não houve interação significativa entre grupos e momentos no tempo do TUG simples $(p=0.678)$, tempo do TUG-DT $(p=0.524)$ e custo da tarefa motora $(p=0.513)$. Não houve diferenças significativas entre grupos e entre momentos em nenhuma variável, embora o GI tenha mostrado uma redução no tempo do TUG-DT após 16 semanas. Conclusão: Embora a hidroterapia não apresentou melhora significativa na mobilidade dos idosos, houve uma redução relevante clinicamente do tempo do TUG-DT, sugerindo continuidade de pesquisas com amostra maior.

Palavras-chave: Idosos; Exercícios aquáticos; Mobilidade; TUG; Dupla-tarefa.

Agradecimentos: Os autores agradecem o apoio da Coordenação de Aperfeiçoamento de Pessoal de Nível Superior - Brasil (CAPES) - Código de Financiamento (001) e o apoio financeiro do CNPq. 